\title{
Generation of three-dimensional versatile vortex linear light bullets
}

\section{(Invited Paper)}

\author{
Han $\mathrm{Li}^{1,3}$, Xin Huang ${ }^{1,7}$, Qian Cao ${ }^{1,4,6}$, Yun Zhao ${ }^{1}$, Peiyun $\mathrm{Li}^{1}$, Chenchen Wan ${ }^{1,5}$, \\ and Andy Chong ${ }^{1,2, *}$ \\ ${ }^{1}$ Department of Electro-Optics and Photonics, University of Dayton, Dayton, OH, 45469, USA \\ ${ }^{2}$ Department of Physics, University of Dayton, Dayton, OH 45469, USA \\ ${ }^{3}$ Image Sensor Group, ON Semiconductor, San Jose, CA 95134, USA \\ ${ }^{4}$ Center for Free-Electron Laser Science, Hamburg 22607, Germany \\ ${ }^{5}$ Deutsches-Elektronen Synchrotron (DESY), Hamburg 22607, Germany \\ ${ }^{6}$ Department of Physics, University of Hamburg, Hamburg 22761, Germany \\ ${ }^{7}$ Intelligent Robot System Department, SIASUN Robot \& Automation Co., Ltd., \\ Shenyang 110168, China \\ *Corresponding author: achong1@udayton.edu
}

Received December 1, 2016; accepted January 23, 2017; posted online February 17, 2017

\begin{abstract}
We generate and measure the versatile vortex linear light bullet, which combines a high-order Bessel beam and an Airy pulse. This three-dimensional optical wave packet propagates without distortion in any medium, while carrying an orbital angular momentum. Its non-varying feature in linear propagation is verified by a threedimensional measurement. Such a novel versatile linear light bullet can be useful in various applications such as micromachining.

OCIS codes: $320.7090,320.7100$.

doi: 10.3788/COL201715.030009.
\end{abstract}

Optical wave packet spreading during propagation is an inherent property of optical waves. Diffraction causes optical beams to spread in transverse directions, while material dispersion leads to temporal pulse broadening in the propagation direction. These effects constrain many applications such as optical tweezers, high-resolution imaging, free-space optical communication, etc. $.-3-3]$.

In contrast to general optical wave packets that spread as they propagate, there are certain optical wave packet shapes that do not spread as they propagate. Such nonvarying wave packets are referred to as localized waves ${ }^{[4]}$. In nonlinear propagation, propagation-invariant wave packets, which are called solitons or solitary waves, exist when the nonlinear effect compensates the dispersion or diffraction effect ${ }^{[5]}$. In one-dimensional (1D) propagation, invariant optical soliton pulses can be formed by balancing self-phase modulation (SPM) and anomalous group velocity dispersion (GVD) ${ }^{[6]}$. Two-dimensional (2D) spatial solitons have been experimentally demonstrated as well by balancing the diffraction and the photorefractive effect ${ }^{[]}$. In addition, 2D spatiotemporal solitons have been experimentally generated in a quadratic nonlinear medium by a cascading quadratic nonlinear effect ${ }^{[8]}$.

However, three-dimensional (3D) spatiotemporal optical solitons created by simultaneously balancing a nonlinear effect with dispersion and diffraction phenomena have not been demonstrated due to an instability issue. Hence, the generation of 3D nondispersive packets has been a great experimental challenge for several decades ${ }^{[9]}$. 3D optical wave pockets that maintain their shapes during propagation by balancing dispersion, diffraction, and various nonlinear effects are referred to as light bullets ${ }^{[9]}$. For instance, light bullets are expected to be ideal units in applications such as optical telecommunications, since it maintains its peak power through a dispersive and diffractive medium $\underline{[10]}$.

In linear propagation, 3D linear light bullets, such as $\mathrm{X}$ waves ( $\mathrm{O}$ waves), have been demonstrated, which are exact propagation-invariant solutions of a wave equation for normal (anomalous) dispersion $\underline{[1,12]}$. An X wave can be understood as a superposition of polychromatic Bessel beams, where the non-diffractive feature is due to the Bessel beam profile, and the non-dispersive feature is realized by a controlled balance between the cone angle dispersion of the Bessel beams and GVD of the material ${ }^{[13}$. The X-wave beam can be understood as a coupling between the spatial and temporal profile of the optical wave packet designed to counterbalance the temporal dispersion with the diffraction effect. Since the nonvarying feature of $\mathrm{X}$ waves relies on the spatiotemporal coupling, it is necessary to form a unique $\mathrm{X}$-wave profile to counterbalance a specific GVD of a medium.

There are novel linear light bullets, which work as localized waves for any combination of GVD and diffraction of a medium, known as Airy function profiles. An Airy function is the only available localized analytic solution of a 1D paraxial wave equation. Therefore, an Airy pulse is the only non-dispersive analytic pulse solution for linear 


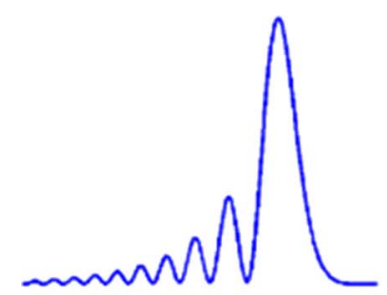

Airy pulse

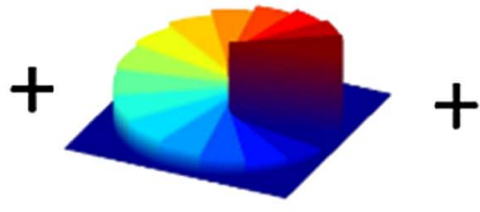

Spiral phase

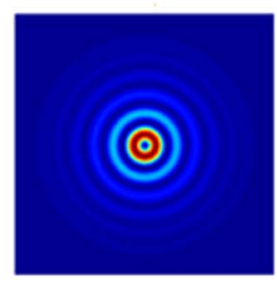

Bessel beam

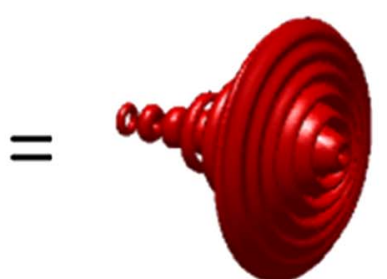

Vortex Airy-Bessel wave packet

Fig. 1. Formation of the vortex Airy-Bessel wave packet.

propagation. Non-diffractive beams can be formed based on Airy profiles such as 1D and 2D Airy beams, which have been recently demonstrated ${ }^{[14]}$. In contrast to traditional non-diffractive beams such as Bessel beams, Airy beams do not rely on the superposition of conically propagating plane waves. Furthermore, Airy waves exhibit additional unique properties such as self-acceleration and self-healing $[15]$.

Airy waves are useful for generating unique linear light bullets. For example, a special linear light bullet is demonstrated by combining an Airy pulse and a Bessel beam. This Airy-Bessel wave packet does not require the balance between the dispersion and the diffraction effects, since Airy pulses and Bessel beams are impervious to dispersion and diffraction, respectively. Since Airy-Bessel wave packets are localized 3D wave packets in any linear media, they are referred to as versatile linear light bullets $\underline{\text { [16] }}$.

In parallel, optical vortices carrying phase singularities with orbital angular momentums have developed as a research topic of a recent interest. Optical vortices have become not only of fundamental scientific interest, but they are also important tools for applications such as optical tweezers, free-space telecommunications, super-resolution optical microscopy, etc. $\frac{[17-19]}{-19}$. The most common intensity profile of vortex beams is the Laguerre-Gaussian (LG) beam that can be generated by a holographic filter ${ }^{[20]}$ or a spiral phase plate $\underline{[21]}$. Stable localized optical vortex solitons that exist in nonlinear media have been demonstrated as well[22].

In this Letter, we experimentally demonstrate a $3 \mathrm{D}$ vortex Airy-Bessel wave packet as a versatile vortex linear light bullet. The vortex Airy-Bessel wave packet contains an Airy pulse in time and a higher-order vortex Bessel beam that carries an orbital angular momentum. It is a $3 \mathrm{D}$ linear light bullet but also carries a phase singularity with an orbital angular momentum. Its non-varying feature in linear propagation is verified by the $3 \mathrm{D}$ intensity and the transverse phase profile measurement. Since this wave packet is invariant with a vortex beam profile for any material, the wave packet has suitable beam shaping with orbital angular momentum with potential applications to laser micromachining $\stackrel{[23]}{ }$. The $3 \mathrm{D}$ paraxial wave equation in linear propagation in a moving reference frame is given as

$$
\frac{1}{2 k} \nabla_{T}^{2} A-\frac{\beta_{2}}{2} \frac{\partial^{2} A}{\partial t^{2}}-i \frac{\partial A}{\partial z}=0,
$$

where $A$ is an envelope function, while the transverse Laplacian is $\nabla_{T}^{2}=\left(\frac{\partial^{2}}{\partial x^{2}}+\frac{\partial^{2}}{\partial y^{2}}\right)$. The first term describes diffraction, and the second term accounts for the dispersion, where $k$ is the wavenumber, and $\beta_{2}$ is the GVD coefficient. Equation (1) admits many versatile propagation-invariant solutions by combining non-diffractive beams such as Bessel, Airy, Matheieu beam, etc., with an Airy pulse. Among such solutions, a propagation-invariant solution of interest is given in $\underline{[24]}$

$$
E(r, z, \tau)=E_{0} A i\left[\frac{\tau}{\tau_{0}}-\frac{\left(\beta_{2}^{2} z^{2}\right)}{4 \tau_{0}^{4}}\right] J_{l}\left(\frac{r}{r_{0}}\right) \exp (i l \phi) .
$$

Equation (2) shows the electric field profile of the vortex Airy-Bessel wave packet. In Eq. (2), $E_{0}$ represents the peak amplitude, while $\tau_{0}$ and $r_{0}$ determine the temporal and the radial width of the wave packet. Since the solution is separable, $\tau_{0}$ and $r_{0}$ are not related (that is $\tau_{0}$ and $r_{0}$ can take any values). $J_{l}(\cdot)$ and $A i(\cdot)$ represent the Bessel and the Airy function, respectively. The exponential term $\exp (i l \phi)$ is the azimuthal phase that describes the vortex structure; $l$ is an integer number, which is referred to as the topological charge. When $|l|>0$, the solution becomes a combination of an Airy pulse and a higher-order Bessel wave packet, which is a vortex beam.

In this experiment, the process of generating a vortex Airy-Bessel wave packet is shown in Fig. 1. First, an Airy pulse with a Gaussian beam profile is formed by a pulse shaping technique ${ }^{[25]}$. Secondly, the Gaussian beam profile is converted into a higher-order Bessel beam by applying an azimuthal and conic phase in sequence ${ }^{[26]}$.

The detailed experimental configuration is shown in Fig. 2. Positively chirped femtosecond pulses from a mode-locked fiber laser are divided into two beam paths. A grating pair is set to compress the chirped pulse into a Fourier transform-limited (TL) pulse, which serves as a probe wave packet for 3D profile measurement (the lower path in Fig. 2). Another beam path (the upper path in Fig. 2) serves as a pulse shaper. By adding a significant cubic spectral phase by the spatial light modulator (SLM) in the pulse shaper, an Airy pulse is formed.

Another SLM is implemented to apply a transverse spiral phase that converts the initial Gaussian beam into an LG vortex beam with a topological charge of one. The LG vortex beam is converted into a higher-order vortex Bessel beam by an axicon, which is a conical lens ${ }^{[26]}$. Since $l=1$, the beam profile is the first-order Bessel beam. 


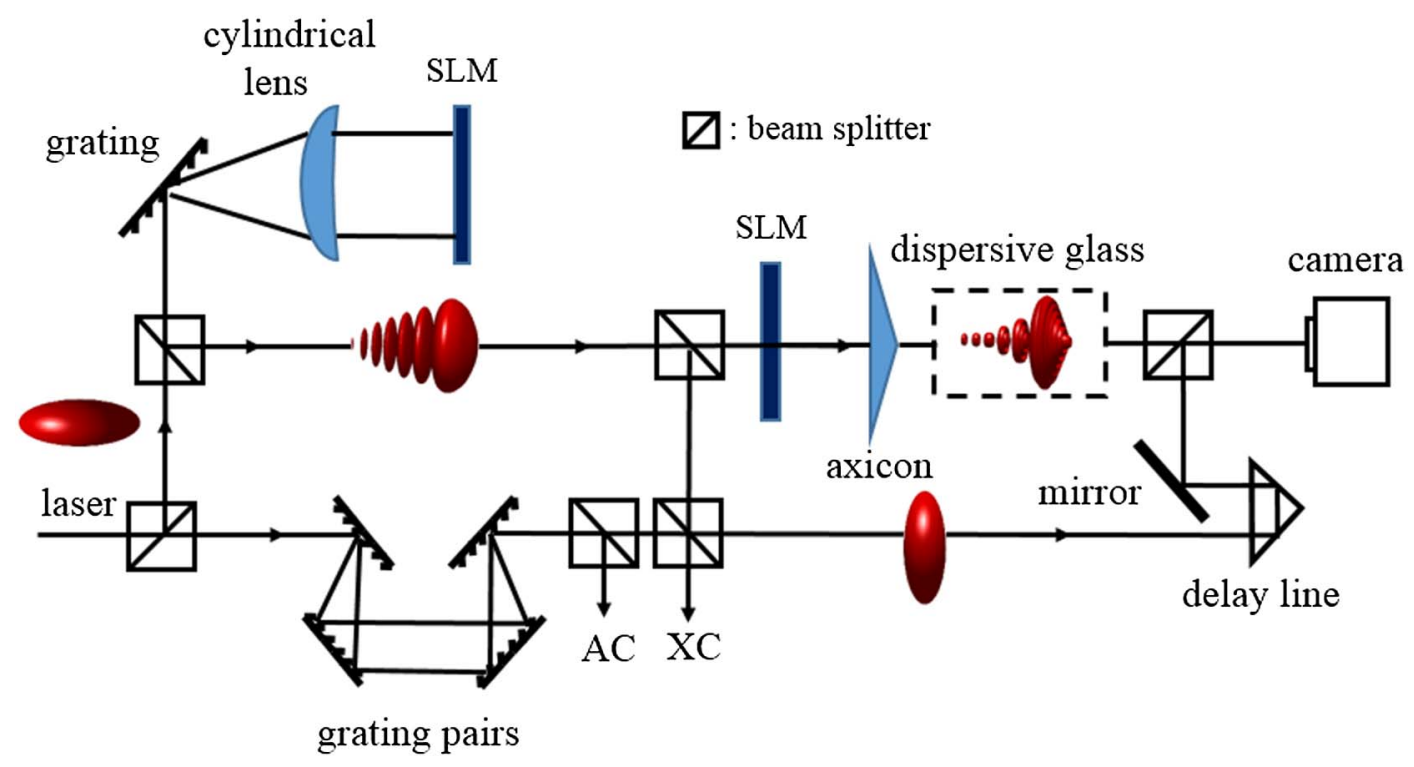

Fig. 2. Experimental setup to generate and measure the vortex Airy-Bessel wave packet. AC, Auto-correlation; XC, Cross-correlation.
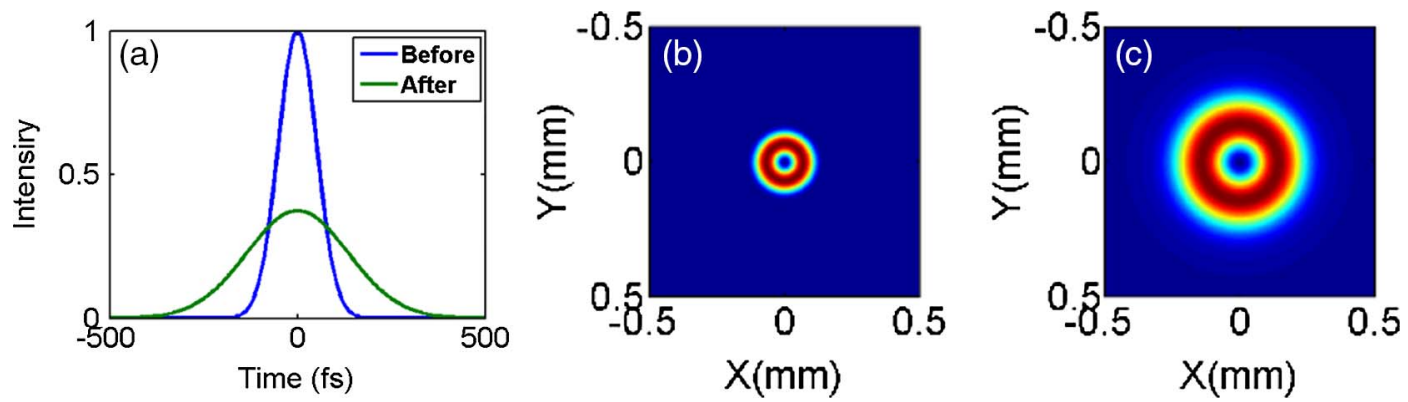

Fig. 3. Dispersion and diffraction effects of the glass rod on (a) a Gaussian pulse and (b) and (c) a ring beam. The duration of the Gaussian beam is the same as the main lobe duration of the Airy pulse. The size of the ring is the same as the first ring of the first-order Bessel beam. The initial ring beam (b) expands $\sim 2$ after the glass rod (c).

The $3 \mathrm{D}$ wave packet profile measurement is performed by overlapping the object wave packet with a timedelayed probe wave packet on a CCD camera. The 3D intensity profile of the object wave packets are retrieved based on techniques proposed in Ref. [27].

In order to demonstrate the non-varying feature of the vortex Airy-Bessel wave packet in linear propagation, a 3D measurement is repeated before and after inserting a dispersive and diffractive element, a 4-in-long SF11 glass rod in the path of the object wave packet. The wave packet experiences a significant dispersion effect, which corresponds to $\sim 2.5$ characteristic dispersion lengths. Theoretically, this dispersion effect is able to broaden a Gaussian pulse with a same duration of the main lobe of the Airy pulse by a factor of $\sim 2.5$ times [Fig. 3(a)]. By inserting the glass rod, it also provides a significant diffraction effect to spread the central ring of the higher-order Bessel beam by a factor of $\sim 2$ in diameter [Figs. $\underline{3(\mathrm{~b})}$ and $\underline{3(\mathrm{c})}$ ].

The measured 3D intensity profile and the azimuthal phase profile of the vortex Airy-Bessel wave packet are shown in Fig. $\underline{4(\mathrm{a})}-\underline{4(\mathrm{c})}$. The 3D measurement clearly shows the Airy pulse profile in time and a higher-order Bessel beam profile of the wave packet. The measured transverse phase profile also clearly indicates a vortex profile with a topological charge of one. The measured duration of the main lobe of the Airy pulse is $147 \mathrm{fs}$, while the measured central ring size of the first-order Bessel beam is $211 \mu \mathrm{m}$ in diameter.

Despite the presence of a medium with strong broadening effects, the 3D measurements [Fig. $\underline{4(\mathrm{~d})}$ and $\underline{4(\mathrm{e})}$ ] reveal that the vortex Airy-Bessel wave packet maintained its $3 \mathrm{D}$ profile. The duration of the main lobe is maintained ( $\sim 135 \mathrm{fs}$ ), while the central ring radius is again maintained $(\sim 194 \mu \mathrm{m}$ in diameter) after propagation through the dispersive and diffractive glass rod. The transverse phase profile [Fig. $\underline{4(\mathrm{f})}$ ] indicates that there are multiple phase defects. However, considering the center portion of the beam, it is clear that azimuthal phase with a topological charge 1 is preserved. The experiment demonstrates that the vortex Airy-Bessel wave packet maintains its localization under the unbalanced dispersion and 
(a)

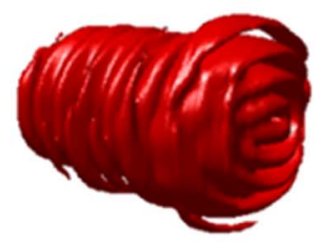

(d)

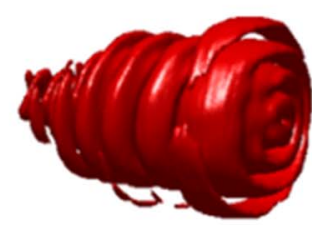

(b)

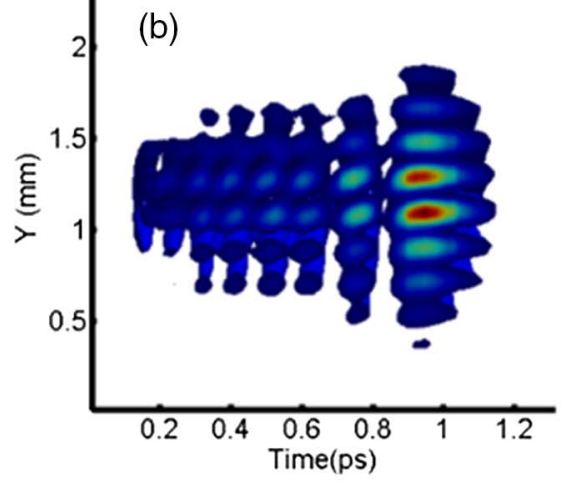

(e)

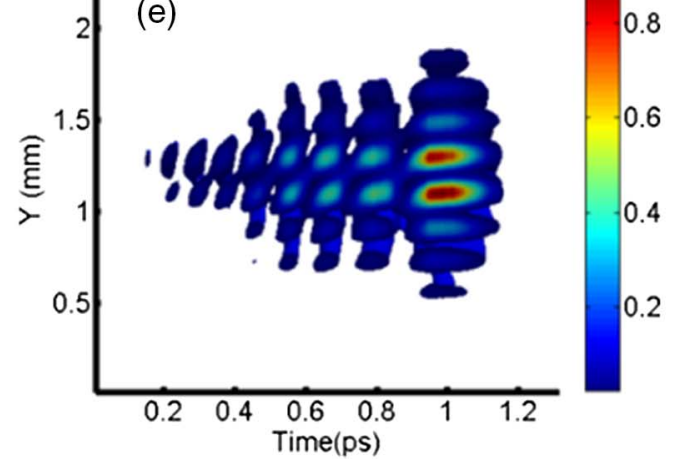

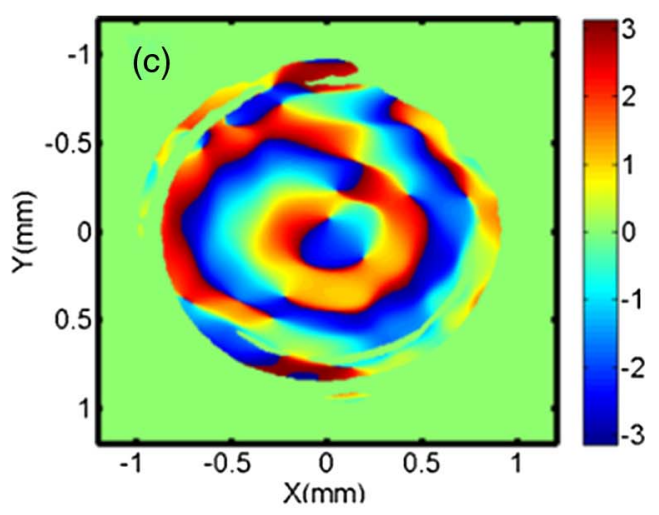

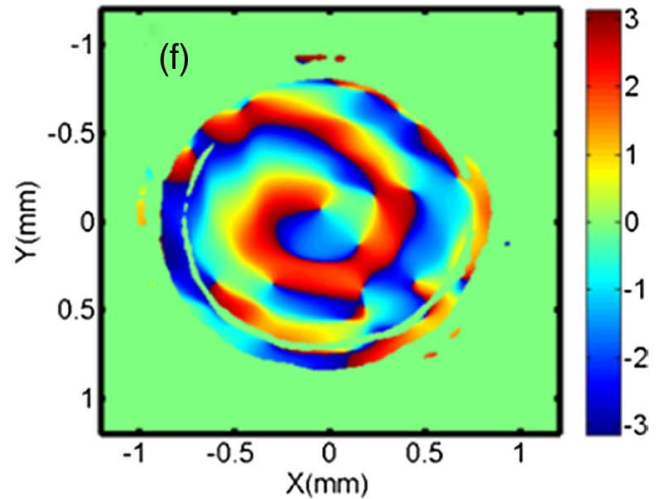

Fig. 4. 3D measurements of the vortex Airy-Bessel wave pocket. The iso-intensity profiles (a) without and (d) with the glass rod. The sagittal intensity profiles (b) without and (e) with the glass. The transverse phase in the range of $(-\pi, \pi)$ profiles (c) without and (f) with the glass rod.

diffraction effects. This verifies that the vortex AiryBessel wave packet serves as a versatile linear light bullet for any linear medium.

In conclusion, we experimentally demonstrate a vortex versatile linear light bullet as a 3D vortex Airy-Bessel wave packet. This wave packet propagates without distortion while carrying an orbital angular momentum. Its propagation-invariant nature is experimentally verified by $3 \mathrm{D}$ diagnostics. The vortex Airy-Bessel wave packet works for any dispersive material as a versatile vortex linear light bullet, which can be widely applied for further applications.

Authors thank professor Joseph Haus at the University of Dayton we provided valuable insight and comments.

\section{References}

1. A. Ashkin, J. M. Dziedzic, J. E. Bjorkholm, and S. Chu, Opt. Lett. 11, 288 (1986).

2. J. W. Goodman, Introduction to Fourier Optics (McGraw-Hill, 1996).

3. J. Jia, C. Zhou, and L. Liu, Opt. Commun. 228, 271 (2003).

4. H. E. Hernandez-Figueroa, M. Zamboni-Rached, and E. Recami, Localized Waves (Wiley, 2008).

5. B. A. Malomed, D. Mihalache, F. W. Wise, and L. Torner, J. Opt. B 7, R53 (2005).

6. L. F. Mollenauer, R. H. Stolen, and J. P. Gordon, Phys. Rev. Lett. 45, 1095 (1980).

7. M. Segev, B. Crosignani, A. Yariv, and B. Fischer, Phys. Rev. Lett. 68, 923 (1992).

8. X. Liu, L. J. Qian, and F. W. Wise, Phys. Rev. Lett. 82, 4631 (1999).
9. Y. Silberberg, Opt. Lett. 15, 1282 (1990).

10. J. W. Moloney, A. C. Newell, and A. B. Aceves, Opt. Quantum Electron. 24, S1269 (1992).

11. J. Lu and J. F. Greenleaf, IEEE Trans. Ultrason. Ferroelec. Freq. Cont. 39, 19 (1992).

12. M. A. Porras and P. Di Trapani, Phys. Rev. E 69, 066606 (2004).

13. H. Sõnajalg, M. Rätsep, and P. Saari, Opt. Lett. 22, 310 (1997).

14. G. A. Siviloglou, J. Broky, A. Dogariu, and D. N. Christodoulides, Phys. Rev. Lett. 99, 213901 (2007).

15. J. Broky, G. A. Siviloglou, A. Dogariu, and D. N. Christodoulides, Opt. Express 16, 12880 (2008).

16. A. Chong, W. H. Renninger, D. N. Christodoulides, and F. W. Wise, Nat. Photon. 4, 103 (2010).

17. D. L. Andrews, A. R. Carter, M. Babiker, and M. Al-Amri, Proc. SPIE 6136, 613103 (2006).

18. G. Gibson, J. Courtial, M. J. Padgett, M. Vasnetsov, V. Pas'ko, S. M. Barnett, and S. Franke-Arnold, Opt. Express 12, 5448 (2004).

19. P. Török and P. R. T. Munro, Opt. Express 12, 3605 (2004).

20. R. Heckenberg, R. McDuff, C. P. Smith, and A. G. White, Opt. Lett. 17, 221 (1992).

21. M. W. Beijersbergen, R. P. C. Coerwinkel, M. Kristensen, and J. P. Woerdman, Opt. Commun. 112, 321 (1994).

22. G. A. Swartzlander, Jr. and C. T. Law, Phys. Rev. Lett. 69, 2503 (1992).

23. R. Oosterbeek, S. Ashforth, O. Bodley, and M. C. Simpson, in CLEO (2016), paper ATh1K.3.

24. G. A. Silviloglou and D. N. Christodoulides, Opt. Lett. 32, 979 (2007). 25. A. M. Weiner, Rev. Sci. Instrum. 71, 1929 (2000).

26. J. Arlt and K. Dholakia, Opt. Commun. 177, 297 (2000).

27. H. Li, I. V. Bazarov, B. M. Dunham, and F. W. Wise, Phys. Rev. ST Accel. Beams 14, 112802 (2011). 